\title{
POSITIVE SOLUTIONS OF BOUNDARY VALUE PROBLEMS FOR $p$-LAPLACIAN FUNCTIONAL DIFFERENCE EQUATIONS
}

\author{
CHANGXIU SONG
}

\begin{abstract}
In this paper, the author studies the boundary value problems of $p$-Laplacian functional difference equation. By using a fixed point theorem in cones, sufficient conditions are established for the existence of the positive solutions.
\end{abstract}

\section{Introduction}

For notation, given $a<b$ in $Z$, we employ intervals to denote discrete sets such as $[a, b]=\{a, a+1, \ldots, b\},[a, b)=\{a, a+1, \ldots b-1\},[a, \infty)=\{a, a+1, \ldots\}$, etc. Let $\tau, T \in Z$ and $0 \leq \tau \leq T$. In this paper, we are concerned with the following $p$-Laplacian difference equation:

$$
\begin{aligned}
& \Delta \phi_{p}(\Delta x(t))+r(t) f\left(x_{t}\right)=0, t \in[0, T] \\
& x_{0}=\psi \in C^{+}, x(0)-B_{0}(\Delta x(0))=0, \Delta x(t+1)=0,
\end{aligned}
$$

where $\phi_{p}(u)$ is the $p$-Laplacian operator, i.e., $\phi_{p}(u)=|u|^{p-2} u, p>1,\left(\phi_{p}\right)^{-1}(u)=\phi_{q}(u)$, $\frac{1}{p}+\frac{1}{q}=1 . \forall t \in Z$, let $x_{t}=x_{t}(k)=x(t+k), k \in[-\tau,-1]$, then $x_{t} \in C$, where $C=C([-\tau,-1], R)$ is a Banach space with the norm $\|\varphi\|_{C}=\max _{k \in[-\tau,-1]}|\varphi|$. Let $C^{+}=$ $\{\varphi \in C: \varphi(k) \geq 0, k \in[-\tau,-1]\}$. As usual, $\Delta$ denotes the forward difference operator defined by $\Delta x(t)=x(t+1)-x(t)$.

We give the following assumptions:

$\left(H_{0}\right) f(\varphi)$ is a nonnegative continuous functional defined on $C^{+}$;

$\left(H_{1}\right) r(t)$ is a nonnegative function defined on $[0, T]$ and $\sum_{t=\tau}^{T} r(t)>0$;

$\left(H_{2}\right) \quad B_{0}: R \rightarrow R$ is continuous and satisfies that there are $\beta \geq \alpha \geq 0$ such that $\alpha s \leq B_{0}(s) \leq \beta s$ for $s \in R^{+}$, where $R^{+}$denotes the set of nonnegative real numbers.

Received February 22, 2006.

2000 Mathematics Subject Classification. 39A05.

Key words and phrases. $p$-Laplacian boundary value problem, functional difference equations, positive solution, fixed point theorem in cones.

Supported by Natural Science Fund of Guangdong Province, China. 
The motivations for the present work stem from many recent investigations in [1-5]. For the continuous or functional case, boundary value prolems analogous to (1.1) are studied by many authors, see, for example [6-11].

The following lemma will be play an important role in the proof of our results and can be found in [12].

Lemma 1.1. Assume that $X$ is a Banach space and $K \subset X$ is a cone in $X ; \Omega_{1}, \Omega_{2}$ are open subsets of $X$, and $0 \in \bar{\Omega}_{1} \subset \Omega_{2}$. Furthermore, let $\Phi: K \cap\left(\bar{\Omega}_{2} \backslash \Omega_{1}\right) \rightarrow K$ be a completely continuous operator satisfying one of the following conditions:

(i) $\|\Phi(x)\| \leq\|x\|, \forall x \in K \cap \partial \Omega_{1} ;\|\Phi(x)\| \geq\|x\|, \forall x \in K \cap \partial \Omega_{2}$;

(ii) $\|\Phi(x)\| \leq\|x\|, \forall x \in K \cap \partial \Omega_{2} ;\|\Phi(x)\| \geq\|x\|, \forall x \in K \cap \partial \Omega_{1}$;

Then there is a fixed point of $\Phi$ in $K \cap\left(\bar{\Omega}_{2} \backslash \Omega_{1}\right)$.

\section{Main results}

We note that $x(t)$ is a solution of (1.1) if and only if

$$
x(t)= \begin{cases}B_{0}\left(\phi_{q}\left(\sum_{n=0}^{T} r(n) f\left(x_{n}\right)\right)\right)+\sum_{m=0}^{t-1} \phi_{q}\left(\sum_{n=m}^{T} r(n) f\left(x_{n}\right)\right), & t \in[0, T+2], \\ \psi, & t \in[-\tau,-1] .\end{cases}
$$

Furthermore, a solution $x(t)$ of (1.1) is called a positive solution, if $x(t)>0$, for $t \in[0, T]$.

We assume that $\bar{x}(t)$ is the solution of BVP (1.1) with $f \equiv 0$. Clearly, it can be expressed as

$$
\bar{x}(t)= \begin{cases}0, & t \in[0, T+2], \\ \psi, & t \in[-\tau,-1] .\end{cases}
$$

Let $x(t)$ be a solution of BVP (1.1) and $y(t)=x(t)-\bar{x}(t)$, noting that $y(t)=x(t)$ for $t \in[0, T+2]$, then we have from (2.1) that

$$
y(t)= \begin{cases}B_{0}\left(\phi_{q}\left(\sum_{n=0}^{T} r(n) f\left(y_{n}+\bar{x}_{n}\right)\right)\right)+\sum_{m=0}^{t-1} \phi_{q}\left(\sum_{n=m}^{T} r(n) f\left(y_{n}+\bar{x}_{n}\right)\right), & t \in[0, T+2], \\ 0, & t \in[-\tau,-1] .\end{cases}
$$

Let $E=\{y:[-\tau, T+2] \rightarrow R\}$ be endowed with the norm $\|y\|=\max _{t \in[-\tau, T+2]}|y(t)|$ and $K=\left\{y \in E: y(t)=0\right.$ for $t \in[-\tau,-1] ; y(t) \geq \frac{1}{T+2+\beta}\|y\|$ for $\left.t \in[0, T+2]\right\}$.

Clearly, $E$ is a Banach space with the norm $\|y\|$ and $K$ is a cone in $E$. If $y(t) \in K$, then $\|y\|=\|y\|_{[0, T+2]}$, where $\|y\|_{[0, T+2]}=\max _{t \in[0, T+2]}|y(t)|$.

Definie $\Phi: K \rightarrow E$ as

$(\Phi y)(t)= \begin{cases}B_{0}\left(\phi_{q}\left(\sum_{n=0}^{T} r(n) f\left(y_{n}+\bar{x}_{n}\right)\right)\right)+\sum_{m=0}^{t-1} \phi_{q}\left(\sum_{n=m}^{T} r(n) f\left(y_{n}+\bar{x}_{n}\right)\right), & t \in[0, T+2] \\ 0, & t \in[-\tau,-1] .\end{cases}$ 
It following from (2.4) that

$$
\begin{aligned}
\|\Phi y\| & =\|\Phi y\|_{[0, T+2]}=(\Phi y)(T+2) \\
& =B_{0}\left(\phi_{q}\left(\sum_{n=0}^{T} r(n) f\left(y_{n}+\bar{x}_{n}\right)\right)\right)+\sum_{m=0}^{t-1} \phi_{q}\left(\sum_{n=m}^{T} r(n) f\left(y_{n}+\bar{x}_{n}\right)\right) \\
& \leq(T+2+\beta) \phi_{q}\left(\sum_{n=0}^{T} r(n) f\left(y_{n}+\bar{x}_{n}\right)\right) .
\end{aligned}
$$

Lemma 2.1. $\Phi(K) \subset K$.

Proof. For $t \in[-\tau,-1],(\Phi y)(t)=0$, and for $t \in[0, T+2]$, we have from (2.4) - 2.5

$$
\begin{aligned}
(\Phi y)(t) & \geq \phi_{q}\left(\sum_{n=0}^{T} r(n) f\left(y_{n}+\bar{x}_{n}\right)\right) \\
& \geq \frac{1}{T+2+\beta}\|\Phi y\|_{[0, T+2]}=\frac{1}{T+2+\beta}\|\Phi y\|,
\end{aligned}
$$

which implies $\Phi(K) \subset K$.

Lemma 2.2. $\Phi: K \rightarrow K$ is completely continuous.

Let

$$
l=\frac{1}{(T+2+\beta) \phi_{q}\left(\sum_{n=0}^{T} r(n)\right)}, \quad M=\frac{T+2+\beta}{\phi_{q}\left(\sum_{n=\tau}^{T} r(n)\right)} .
$$

Theorem 2.1. BVP (1.1) has at least a positive solution if one of the following conditions is satisfied:

$$
\begin{aligned}
& \left(H_{3}\right) \lim _{\|\varphi\|_{C} \downarrow 0} \sup \frac{f(\varphi)}{\|\varphi\|_{C}^{p-1}}<l^{p-1}, \lim _{\|\varphi\|_{C} \uparrow \infty} \inf \frac{f(\varphi)}{\|\varphi\|_{C}^{p-1}}>M^{p-1}, \psi(t) \equiv 0, t \in[-\tau,-1] ; \\
& \left(H_{4}\right) \lim _{\|\varphi\|_{C} \downarrow 0} \inf \frac{f(\varphi)}{\|\varphi\|_{C}^{p-1}}>M^{p-1}, \lim _{\|\varphi\|_{C} \uparrow \infty} \sup \frac{f(\varphi)}{\|\varphi\|_{C}^{p-1}}<l^{p-1} .
\end{aligned}
$$

Proof. Suppose that $\left(H_{3}\right)$ is satisfied. By $\psi(t) \equiv 0, t \in[-\tau,-1]$, we know $\bar{x}_{n} \equiv 0$, $n \in[0, T+2]$. Since $\lim _{\|\varphi\|_{C} \downarrow 0} \frac{f(\varphi)}{\|\varphi\|_{C}^{p-1}}<l^{p-1}$, there is a $\rho_{1}>0$ such that

$$
f(\varphi) \leq\left(l\|\varphi\|_{C}\right)^{p-1}, \quad\|\varphi\|_{C} \leq \rho_{1} .
$$

For any $y \in K$ with $\|y\|=\rho_{1}$, we deduce that $\left\|y_{n}\right\|_{C} \leq \rho_{1}$ for $n \in[0, T+2]$ and have from 2.5

$$
\begin{aligned}
\|\Phi y\| & \leq(T+2+\beta) \phi_{q}\left(\sum_{n=0}^{T} r(n) f\left(y_{n}\right)\right) \\
& \leq l(T+2+\beta)\|y\| \phi_{q}\left(\sum_{n=0}^{T} r(n)\right)
\end{aligned}
$$




$$
=\|y\| \text { and } y \in K \cap \partial \Omega_{\rho_{1}},
$$

where $\Omega_{\rho_{1}}=\left\{y \in K:\|y\|<\rho_{1}\right\}$.

On the other hand, since $\lim _{\|\varphi\|_{C} \uparrow \infty} \inf \frac{f(\varphi)}{\|\varphi\|_{C}^{p-1}}>M^{p-1}$, there exists a $\rho_{2}>\rho_{1}$ such that

$$
f(\varphi) \geq\left(M\|\varphi\|_{C}\right)^{p-1}, \quad\|\varphi\|_{C} \geq \frac{\rho_{2}}{T+2+\beta} .
$$

Define $\Omega_{\rho_{2}}=\left\{y \in K:\|y\|<\rho_{2}\right\}$. For $y \in K$ with $\|y\|=\rho_{2}$, we have

$$
y(t) \geq \frac{1}{T+2+\beta}\|y\|, \quad t \in[0, T+2],
$$

and

$$
\left\|y_{n}\right\|_{C} \geq \frac{1}{T+2+\beta}\|y\|
$$

Thus, we have from (2.5) -2.8

$$
\begin{aligned}
\|\Phi y\| & =\|\Phi y\|_{[0, T+2]}=(\Phi y)(T+2) \\
& \geq \sum_{m=0}^{T+1} \phi_{q}\left(\sum_{n=m}^{T} r(n) f\left(y_{n}\right)\right) \\
& \geq \sum_{m=\tau}^{T+1} \phi_{q}\left(\sum_{n=m}^{T} r(n)\left(M\left\|y_{n}\right\|_{C}\right)^{p-1}\right) \\
& \geq \frac{M}{T+2+\beta}\|y\| \phi_{q}\left(\sum_{n=\tau}^{T} r(n)\right) \\
& =\|y\| \text { for } y \in K \cap \partial \Omega_{\rho_{2}} .
\end{aligned}
$$

According to the first part of Lemma 1.1, it follows that $\Phi$ has a fixed point $y \in$ $K \cap\left(\bar{\Omega}_{2} \backslash \Omega_{1}\right)$ such that

$$
0<\rho_{1} \leq\|y\|=\|y\|_{[0, T+2]} \leq \rho_{2} .
$$

Now, suppose that $\left(H_{4}\right)$ is satisfied. Since $\lim _{\|\varphi\|_{C} \downarrow 0} \inf \frac{f(\varphi)}{\|\varphi\|_{C}^{p-1}}>M^{p-1}$, there exists a $\rho_{1}>0$ such that

$$
f(\varphi) \geq\left(M\|\varphi\|_{C}\right)^{p-1}, \quad\|\varphi\|_{C} \leq \rho_{1} .
$$

For $y \in K$ with $\|y\|=\rho_{1}$, we have $\left\|y_{n}\right\|_{C} \leq \rho_{1}$ for $n \in[\tau, T+2]$. Furthermore, by asimilar argument as (2.8), we have

$$
\|y\| \geq\left\|y_{n}\right\|_{C} \geq \frac{1}{T+2+\beta}\|y\|, \quad n \in[\tau, T+2] .
$$


For $n \in[\tau, T+2]$, we have $\bar{x}_{n}=0$. Thus, we obtain

$$
\begin{aligned}
\|\Phi y\| & =\|\Phi y\|_{[0, T+2]}=(\Phi y)(T+2) \\
& \geq \sum_{m=0}^{T+1} \phi_{q}\left(\sum_{n=m}^{T} r(n) f\left(y_{n}\right)\right) \\
& \geq \sum_{m=\tau}^{T+1} \phi_{q}\left(\sum_{n=m}^{T} r(n)\left(M\left\|y_{n}\right\|_{C}\right)^{p-1}\right) \\
& \geq \frac{M}{T+2+\beta}\|y\| \phi_{q}\left(\sum_{n=\tau}^{T} r(n)\right) \\
& =\|y\| \text { for } y \in K \cap \partial \Omega_{\rho_{1}} .
\end{aligned}
$$

On the other hand, since $\lim _{\|\varphi\|_{C} \uparrow \infty} \sup \frac{f(\varphi)}{\|\varphi\|_{C}^{p-1}}<l^{p-1}$, there exists $N>\max \left\{\rho_{1}\right.$, $\left.\max _{k \in[-\tau,-1]}|\psi(k)|\right\}$ such that

$$
f(\varphi)<\left(l\|\varphi\|_{C}\right)^{p-1}, \quad\|\varphi\|_{C}>N .
$$

Choose a positive constant $\rho_{2}$ such that

$$
\rho_{2}>\rho_{1}+l^{-1} \max \left\{f^{q-1}(\varphi): 0 \leq\|\varphi\|_{C} \leq N+\|\bar{X}\|\right\} .
$$

For $y \in K,\|y\|=\rho_{2}$, we have from the facts: $\bar{x}(t) \geq 0, y(t) \geq 0$ for $t \in[-\tau, T+2]$, that for $n \in[0, T]$

$$
\begin{aligned}
& \left\|y_{n}+\bar{x}_{n}\right\|_{C} \geq\left\|y_{n}\right\|_{C}>N, \quad\left\|y_{n}\right\|_{C}>N, \\
& \left\|y_{n}+\bar{x}_{n}\right\|_{C} \leq\left\|y_{n}\right\|_{C}+\left\|\bar{x}_{n}\right\| \leq N+\|\bar{x}\|, \quad\left\|y_{n}\right\|_{C} \leq N
\end{aligned}
$$

Thus, we have

$$
\begin{aligned}
\|\Phi y\| & =\|\Phi y\|_{[0, T+2]}=(\Phi y)(T+2) \\
& =B_{0}\left(\phi_{q}\left(\sum_{n=0}^{T} r(n) f\left(y_{n}+\bar{x}_{n}\right)\right)\right)+\sum_{m=0}^{T+1} \phi_{q}\left(\sum_{n=m}^{T} r(n) f\left(y_{n}+\bar{x}_{n}\right)\right) \\
& \leq(T+2+\beta) \phi_{q}\left(\sum_{n=0}^{T} r(n) f\left(y_{n}+\bar{x}_{n}\right)\right) \\
& =(T+2+\beta) \phi_{q}\left(\sum_{\left\|y_{n}\right\|_{C}>N} r(n) f\left(y_{n}+\bar{x}_{n}\right)+\sum_{\left\|y_{n}\right\|_{C}<N} r(n) f\left(y_{n}+\bar{x}_{n}\right)\right) \\
& \leq(T+2+\beta) \max \left\{l\|y\|, \max \left\{f^{q-1}(\varphi): 0 \leq\|\varphi\|_{C} \leq N+\| \bar{x} \mid\right\}\right\} \phi_{q}\left(\sum_{n=0}^{T} r(n)\right) \\
& =l^{-1} \max \left\{l\|y\|, \max \left\{f^{q-1}(\varphi): 0 \leq\|\varphi\|_{C} \leq N+\|\bar{x}\|\right\}\right\} \\
& \leq \rho_{2}=\|y\| \text { for } y \in K \cap \partial \Omega_{\rho_{2}} .
\end{aligned}
$$


By the second prat of Lemma 1.1, it follows that $\Phi$ has a fixed point $y \in K \cap\left(\bar{\Omega}_{2} \backslash \Omega_{1}\right)$ such that

$$
0<\rho_{1}<\|y\|=\|y\|_{[0, T+2]} \leq \rho_{2}
$$

Suppose that $y$ is the fixed point of $\Phi$ in $K \cap\left(\bar{\Omega}_{2} \backslash \Omega_{1}\right)$, then $x=y+\bar{x}$ is a positive solution of BVP (1.1).

In what follows, we shall consider the existence of twin positive solutions for BVP (1.1).

Theorem 2.2. If the following conditions are satisfied:

$\left(H_{5}\right) \lim _{\|\varphi\|_{C} \downarrow 0} \inf \frac{f(\varphi)}{\|\varphi\|_{C}^{p-1}}>M^{p-1} ; \lim _{\|\varphi\|_{C} \uparrow \infty} \inf \frac{f(\varphi)}{\|\varphi\|_{C}^{p-1}}>M^{p-1} ;$

$\left(H_{6}\right)$ there exists a $p_{1}>0$ such that for $\forall 0 \leq\|\varphi\|_{C} \leq p_{1}+p_{0}$, one has $f(\varphi) \leq\left(l p_{1}\right)^{p-1}$, where $p_{0}=\max _{k \in[-\tau,-1]}|\psi(k)|$.

Then $B V P$ (1.1) has at least two positive solutions $x_{1}, x_{2}$ such that $0<\left\|x_{1}\right\|_{[0, T+2]}<$ $p_{1}<\left\|x_{2}\right\|_{[0, T+2]}$.

Proof. By $\left(H_{5}\right)$, there exists a $r: 0<r<p_{1}$ such that

$$
f(\varphi) \geq\left(M\|\varphi\|_{C}\right)^{p-1}, \quad\|\varphi\|_{C} \leq r .
$$

For $y \in K,\|x\|=r$, we have

$$
r \geq\left\|y_{n}\right\|_{C} \geq \frac{1}{T+2+\beta}\|y\|=\frac{r}{T+2+\beta}, \quad n \in[\tau, T+1] .
$$

Therefore we obtain a analogous inequality:

$$
\|\Phi(y)\| \geq\|y\| \text { for } y \in K \cap \partial \Omega_{r},
$$

where $\Omega_{r}=\{y \in K:\|y\|<r\}$.

On the other hand, we have from $\left(H_{5}\right)$ that there exists a $R>p_{1}$ such that

$$
f(\varphi) \geq\left(M\|\varphi\|_{C}\right)^{p-1}, \quad\|\varphi\|_{C} \geq \frac{R}{T+2+\beta} .
$$

For $y \in K,\|y\|=R$, we have a analogous result to (2.8):

$$
\left\|y_{n}\right\|_{C} \geq \frac{1}{T+2+\beta}\|y\|=\frac{R}{T+2+\beta} \text { for } n \in[\tau, T+1] .
$$

Furthermore, we have $\|\Phi(y)\| \geq\|y\|$ for $y \in K \cap \partial \Omega_{R}$, where $\Omega_{R}=\{y \in K:\|y\|<R\}$.

Now, by $\left(H_{6}\right)$, for $\forall y \in K$ with $\|y\|=p_{1}$ one has

$$
\begin{aligned}
\|\Phi y\| & =\|\Phi y\|_{[0, T+2]}=(\Phi y)(T+2) \\
& =B_{0}\left(\phi_{q}\left(\sum_{n=0}^{T} r(n) f\left(y_{n}+\bar{x}_{n}\right)\right)\right)+\sum_{m=0}^{T+1} \phi_{q}\left(\sum_{n=m}^{T} r(n) f\left(y_{n}+\bar{x}_{n}\right)\right) \\
& \leq(T+2+\beta) l p_{1} \phi_{q}\left(\sum_{n=0}^{T} r(n)\right) \\
& =p_{1}=\|y\| .
\end{aligned}
$$


According to Lemma 1.1, it follows that $\Phi$ has two fixed points $y_{1}, y_{2}$ such that $y_{1} \in$ $K \cap \bar{\Omega}_{p_{1}} \backslash \Omega_{r}, y_{2} \in K \cap \bar{\Omega}_{R} \backslash \Omega_{p_{1}}$, where $\Omega_{p_{1}}=\left\{y \in K:\|y\|<p_{1}\right\}$, that is $0<$ $\left\|y_{1}\right\|<p_{1}<\left\|y_{2}\right\|$. Since $y_{i} \in K$, we have $y_{i}(t)>0, \forall t \in[0, T+2], i=1,2$. Let $x_{1}=y_{1}+\bar{x}, x_{2}=y_{2}+\bar{x}$, then $x_{1}, x_{2}$ are positive solutions of BVP (1.1) satisfying $0<\left\|x_{1}\right\|_{[0, T+2]}<p_{1}<\left\|x_{2}\right\|_{[0, T+2]}$.

Theorem 2.3. If the following conditions are satisfied:

$\left(H_{7}\right) \lim _{\|\varphi\|_{C} \downarrow 0} \sup \frac{f(\varphi)}{\|\varphi\|_{C}^{p-1}}<l^{p-1}, \lim _{\|\varphi\|_{C} \uparrow \infty} \sup \frac{f(\varphi)}{\|\varphi\|_{C}^{p-1}}<l^{p-1}, \psi(t) \equiv 0, t \in[-\tau,-1] ;$

$\left(H_{8}\right)$ there exists a $p_{2}>0$ such that for $\forall \frac{p_{2}}{T+2+\beta} \leq\|\varphi\|_{C} \leq p_{2}$, one has

$$
f(\varphi) \geq\left(\frac{M p_{2}}{T+2+\beta}\right)^{p-1}
$$

Then BVP (1.1) has at least two posivive solutions $x_{1}$, $x_{2}$ satisfying $0<\left\|x_{1}\right\|_{[0, T+2]}<$ $p_{2}<\left\|x_{2}\right\|_{[0, T+2]}$.

The proof of Theorem 2.3 is analogous to Theorem 2.2 and thus is omitted.

The following Corollaries are obvious.

Corollary 2.1. BVP (1.1) has at least a positive solution if one of the following conditions is satisfied:

$\left(H_{3}^{\prime}\right) \lim _{\|\varphi\|_{C} \downarrow 0} \sup \frac{f(\varphi)}{\|\varphi\|_{C}^{p-1}}=0, \lim _{\|\varphi\|_{C} \uparrow \infty} \inf \frac{f(\varphi)}{\|\varphi\|_{C}^{p-1}}=+\infty, \phi(t) \equiv 0, t \in[-\tau,-1] ;$

$\left(H_{4}^{\prime}\right) \lim _{\|\varphi\|_{C} \downarrow 0} \inf \frac{f(\varphi)}{\|\varphi\|_{C}^{p-1}}=+\infty, \lim _{\|\varphi\|_{C} \uparrow \infty} \sup \frac{f(\varphi)}{\|\varphi\|_{C}^{p-1}}=0$.

Corollary 2.2. If the following conditions are satisfied:

$\left(H_{5}^{\prime}\right) \lim _{\|\varphi\|_{C} \downarrow 0} \inf \frac{f(\varphi)}{\|\varphi\|_{C}^{p-1}}=+\infty ; \lim _{\|\varphi\|_{C} \uparrow \infty} \inf \frac{f(\varphi)}{\|\varphi\|_{C}^{p-1}}=+\infty ;$

$\left(H_{6}\right)$ there exists a $p_{1}>0$ such that for $\forall 0 \leq\|\varphi\|_{C} \leq p_{1}+p_{0}$, one has $f(\varphi) \leq\left(l p_{1}\right)^{p-1}$, where $p_{o}=\max _{k \in[-\tau,-1]}|\psi(k)|$.

Then BVP (1.1) has got at least two positive solutions $x_{1}$, $x_{2}$ satisfying $0<\left\|x_{1}\right\|_{[0, T+2]}<$ $p_{1}<\left\|x_{2}\right\|_{[0, T+2]}$.

Corollary 2.3. If the following conditions are satisfied:

$\left(H_{7}^{\prime}\right) \lim _{\|\varphi\|_{C} \downarrow 0} \sup \frac{f(\varphi)}{\|\varphi\|_{C}^{p-1}}=0, \lim _{\|\varphi\|_{C} \uparrow \infty} \sup \frac{f(\varphi)}{\|\varphi\|_{C}^{p-1}}=0, \phi(t) \equiv 0, t \in[-\tau,-1] ;$

$\left(H_{8}\right)$ there exists a $p_{2}>0$ such that for $\forall \frac{p_{2}}{T+2+\beta} \leq\|\varphi\|_{C} \leq p_{2}$, one has $f(\varphi) \geq$ $\left(\frac{M p_{2}}{T+2+\beta}\right)^{p-1}$.

Then BVP (1.1) has got at least two positive solutions $x_{1}$, $x_{2}$ satisfying $0<\left\|x_{1}\right\|_{[0, T+2]}<$ $p_{2}<\left\|x_{2}\right\|_{[0, T+2]}$. 


\section{Example}

Example 3.1. Consider BVP:

$$
\begin{aligned}
& \Delta \phi_{p}(\Delta x(t))+r(t) x^{3}(t-1)=0, \quad t \in[0, T], \\
& x(-1)=0 ; \quad x(0)-B_{0}(\Delta x(0))=0 ; \quad x(T+1)=X(T+2),
\end{aligned}
$$

where $\tau=1<T, 1<p<4, f(\varphi)=\varphi^{3}(-1), r(t)$ satisfies $\left(H_{1}\right)$. As $\varphi \in C^{+},\|\varphi\|_{C} \rightarrow 0$ we have that

$$
\frac{f(\varphi)}{\|\varphi\|_{C}^{p-1}}=\frac{\varphi^{3}(-1)}{\|\varphi\|_{C}^{p-1}}=\frac{\|\varphi\|_{C}^{3}}{\|\varphi\|_{C}^{p-1}}=\|\varphi\|_{C}^{4-p} \rightarrow 0 .
$$

That is to say that $\lim _{\|\varphi\|_{C} \downarrow 0} \sup \frac{f(\varphi)}{\|\varphi\|_{C}^{p-1}}=0$ holds.

On the other hand, suppose that $\varphi \in C^{+}$, then $\|\varphi\|_{C}=\varphi(-1)$, thus, as $\|\varphi\|_{C} \rightarrow \infty$ we get

$$
\frac{f(\varphi)}{\|\varphi\|_{C}^{p-1}}=\frac{\varphi^{3}(-1)}{\|\varphi\|_{C}^{p-1}}=\|\varphi\|_{C}^{4-p} \rightarrow+\infty .
$$

That is to say that $\lim _{\|\varphi\|_{C} \downarrow 0} \inf \frac{f(\varphi)}{\|\varphi\|_{C}^{p-1}}=\infty$ holds.

According to Corollary 2.1, it follows that BVP (3.1) has at least a positive solution $y(t)$.

Example 3.2. Consider BVP:

$$
\begin{aligned}
& \Delta \phi_{p}(\Delta x(t))+r\left[x^{\frac{1}{9}}(t-1)+x^{\frac{1}{3}}(t-1)\right]=0, \quad t \in[0, T] \\
& x(t)=\psi(t) ; \quad t=-1 ; \quad x(0)-B_{0}(\Delta x(0))=0 ; \quad x(T+1)=X(T+2),
\end{aligned}
$$

where $\tau=1<T, r>0$ is a constant. $\psi(t) \geq 0,\|\psi\|_{C}=m_{0}=|\psi(-1)|>0, p=\frac{7}{6}$, $q=7, f(\varphi)=\varphi^{\frac{1}{9}}(-1)+\varphi^{\frac{1}{3}}(-1)$. Suppost that $\varphi \in C^{+}$, then $\|\varphi\|_{C}=\varphi(-1)$, thus, as $\|\varphi\|_{C} \rightarrow 0$ or $\|\varphi\|_{C} \rightarrow+\infty$ we get

$$
\frac{f(\varphi)}{\|\varphi\|_{C}^{p-1}}=\frac{\varphi^{1 / 9}(-1)+\varphi^{1 / 3}(-1)}{\|\varphi\|_{C}^{p-1}}=\|\varphi\|_{C}^{\frac{10-9 p}{9}}+\|\varphi\|_{C}^{\frac{4-3 p}{3}} \rightarrow+\infty .
$$

We deduce that

$$
l=\frac{1}{(T+2+\beta) \phi_{q}\left(\sum_{n=0}^{T} r(n)\right)}=\frac{1}{(T+2+\beta) \phi_{q}((T+1) r)}=\frac{1}{(T+2+\beta)(T+1)^{6} r^{6}},
$$

then for $\forall m>0$ and $0 \leq\|\varphi\|_{C} \leq m+m_{0}$, one has

$$
0 \leq f(\varphi) \leq\left(m+m_{0}\right)^{\frac{1}{9}}+\left(m+m_{0}\right)^{\frac{1}{3}}=\left(m+m_{0}\right)^{\frac{1}{9}}\left(m^{1-p}+\frac{\left(m+m_{0}\right)^{\frac{2}{9}}}{m^{p-1}}\right) m^{p-1} .
$$


Define $H(m)=\left(m+m_{0}\right)^{\frac{1}{9}}\left(m^{1-p}+\frac{\left(m+m_{0}\right)^{\frac{2}{9}}}{m^{p-1}}\right)$, then

$$
\lim _{m \rightarrow 0} H(m)=+\infty, \quad \lim _{m \rightarrow+\infty} H(m)=+\infty .
$$

Suppose that, $r, T$ and $m_{0}$ satisfy

$$
\left(2 m_{0}\right)^{\frac{1}{9}}\left(m_{0}^{-\frac{1}{6}}+2^{\frac{2}{9}} m_{0}^{\frac{1}{18}}\right)<\frac{1}{r(T+1)(T+2+\beta)^{\frac{1}{6}}}=l^{p-1},
$$

then $H\left(m_{0}\right)=\left(2 m_{0}\right)^{\frac{1}{9}}\left(m_{0}^{-\frac{1}{6}}+2^{\frac{2}{9}} m_{0}^{\frac{1}{18}}\right)<l^{p-1}$ holds. By the continuity of $H(m)$ and (3.3), we can found a $m>0$ (for example $m=m_{0}$ ) such that $f(\varphi)<H(m) m^{p-1}<$ $(l m)^{p-1}$ for $0 \leq\|\varphi\|_{C} \leq m+m_{0}$. By the Corollary 2.2, we know that BVP (3.2) has at least two positive solutions.

\section{References}

[1] R. P. Agarwal and J. Henderson, Positive solutions and nonlinear eigenvalue problems for third-order difference equationsm, Comput. Math. Appl. 36(1998), 347-355.

[2] Y. J. Liu and W. G. Ge, Twin positive solutions of boundary value problems for finite difference equations with p-Laplacian operator, J. Math. Anal. Appl. 278(2003), 551-561.

[3] A. Cabada, Extremal solutions for the difference $\phi$-Laplacian problem with nonlinear functional boundary conditions, Comput. Math. Appl. 42(2001), 593-601.

[4] J. Henderson, Positive solutions for nonlinear difference equations, Nonlinear Stud. 4(1997), $29-36$.

[5] P. X. Weng and Z. H. Guo, Existence of positive solutions for nonlinear functional difference equation with p-Laplacian operator, Acta Mathematica Sinica 49(2006), 187-194.

[6] L. H. Erbe and H. Y. Wang, On the existence of positive solutions of ordinary differential equations, Proc. Amer. Math. Soc. 120(1994), 743-748.

[7] F. H. Wong, Existence of positive solutions for $m$-Laplacian Bvps., Appl. Math. Letters 12(1999), 11-17.

[8] R. Manasevich and F. Zanolin, Time-mapping and multiplicity of solutions for the onedimensional p-Laplacians, Nonlinear Analysis 21(1993), 269-291.

[9] X. M. He and W. G. Ge, Existence of positive solutions for the one-dimensional p-Laplacian equations, Acta Mathematica Sinica 46(2003), 805-810.

[10] P. X. Weng and D. Q. Jing, Existence of positive solutions for boundary value problem of second-order FDE, Computers and Mathematics with Applications 37(1999), 1-9.

[11] L. H. Erbe and Q. K. Kong, Boundary value problems for singular second-order functional differential equations, J. Comput. Appl. Math. 53(1994), 377-388.

[12] M. A. Krasnosel'skii, Positive Solutions of Operator Equations, Noordhoof, Groningen, 1964.

School of Applied Mathematics, Guangdong University of Technology, Guangzhou, 510006, China.

E-mail: scx168@sohu.com 